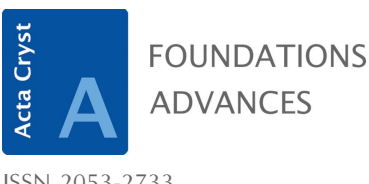

ISSN 2053-2733
Keywords: book review; electrons in solids; condensed matter physics.

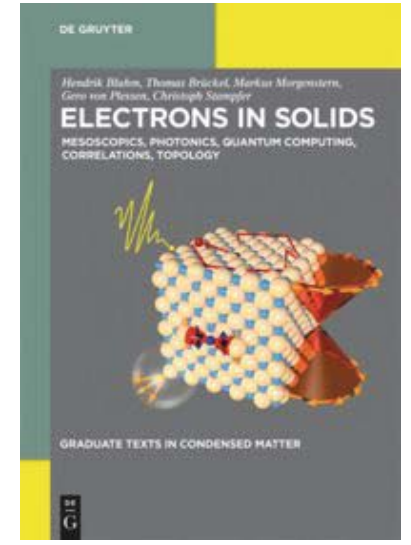

(C) 2021 International Union of Crystallography

\section{Electrons in Solids. Mesoscopics, Photonics, Quantum Computing, Correlations, Topology. By Hendrik Bluhm, Thomas Brückel, Markus Morgenstern, Gero Plessen and Christoph Stampfer. De Gruyter, 2019. Paperback, pp. xii+393. Price EUR 59.95, USD 68.99, GBP 54.50. ISBN 978-3-11-043831-4.}

\author{
Malte Henkel*
}

Université de Lorraine, CNRS, LPCT, Nancy, France. *Correspondence e-mail: malte.henkel@univ-lorraine.fr

Solid-state physics is one of the central fields for the application of quantum mechanics, and from these applications stem many important technologies for innovations on an industrial scale. The present textbook aims at preparing master students of physics for modern topics of research, by going beyond the more classical fields of cooperative phenomena such as ferromagnetism or superconductivity. The book contains the lectures of five recognized experts in the field and the book's subtitle, namely 'Mesoscopics, photonics, quantum computing, correlations, topology', indicates an innovative approach with respect to a more traditional programme. The authors wisely restrict themselves to a few well chosen examples instead of aiming at an encyclopaedic exposition (which would be almost impossible anyway and would almost certainly become rapidly obsolete). Their emphasis is on teaching the physical understanding such that the reader will be enabled to select an adequate level of description and complexity for the specific problem at hand. The result of these conscious efforts is a very readable text on the physical foundations of solid-state physics which will likely serve as an introductory text for advanced students and young, early-career researchers for some time to come.

The main intended readership of this book is experimental physicists, from the master's level onward. However, theoreticians will also benefit from the simple explanations of basic facts and the content of this book will provide useful background for more mathematically oriented studies. Clearly, this is not the place to look up properties of specific theoretical models. On the other hand, the conception of the book should also make it useful to practitioners from neighbouring fields, provided these have the necessary knowledge of quantum mechanics.

The book is organized such that complexity and the importance of quantum effects increase from one chapter to the next, as the following short summary should illustrate. The first chapter, written by C. Stampfer, treats mesoscopic physics and transport. For beginners, the short review on two-dimensional electron systems and how to make them will be useful. A semi-classical treatment focused on phase coherence, and the exemplification through the Aharonov-Bohm effect, is sufficient to proceed from the Drude model to ballistic transport, the Landauer-Buettiker formula and Anderson localization. The second chapter, written G. v. Plessen, discusses optical properties of electrons in solids, mainly for semiconductors and metals. This can to a large extent still be treated using only classical electrodynamics, for example for light absorption in solids. However, laser physics does require a coherent quantum treatment, and this chapter focuses on inter-band transitions, important for photodiodes, photovoltaic cells or light-emitting diodes. Excitons and luminescence arising from intra-band transitions are treated as well and the most common spectroscopic experimental methods are described. The third chapter, written by H. Bluhm, treats the very timely topic of quantum computing, considered by many as the most important emerging application of quantum mechanics. This requires analysis of the quantum dynamics of superposition states, to be used for the solution of huge computational problems of which certain classes can be treated more efficiently with quantum computing than with classical computers. The essential ingre- 
dient is the quantum coherent description of the degrees of freedom involved, which naturally offers an enormous potential for parallelizations in computations. The chapter presents an introduction to these concepts and also shows how the precise experimental control of this quantum superposition leads to a physical understanding when this coherence breaks down, that is de-coherence. Of course, qubits and their experimental realizations are discussed in broad detail. The fourth chapter, written by T. Brückel, studies the quantum interactions between electrons, a notoriously hard problem indeed! One reason for this is the long-range nature of the interactions arising. Starting from a simple mean-field model based on the Schrödinger equation, the tight-binding model before the correlation terms are added, the mechanism of band-gap opening is elucidated. The Hubbard model is introduced to represent the second-quantized electron wavefunctions and their various exchange interactions. The effects of strongly correlated electrons arise for example in hightemperature superconductors, in colossal magneto-resistance, the magneto-caloric effect or metal-insulator transitions. Systems with negative thermal expansion show that these effects occur far from equilibrium and therefore cannot be predicted from the equilibrium quantum Hamiltonian alone. Hence, the study of strong quantum correlations may pave the way towards the fabrication of new materials with unconventional properties. Finally, the fifth chapter, written by M.
Morgenstern, discusses itinerant electrons, their interactions and related questions on their topology. The Peierls transition is the starting point for further developments. Many emergent phenomena exist that require the introduction of 'exotic' quasi-particles with non-standard properties such as a fractional electric charge or 'fractional' statistics, relevant for example in the fractional quantum Hall effect. In order to familiarize the reader with this less commonly used tool, a detailed presentation of the quantum Hall effect in terms of topology is given. Of course, a timely presentation cannot leave out topological insulators, which are treated at length.

The text is well illustrated by many figures, which are easy to localize with the help of a detailed table of figures at the end of the book. Furthermore, a long list of symbols helps to resolve ambiguities in the notation and the index is a decent length (a little more than 6 pages). I would have liked to see a dictionary added, with the technical terms defined in the text translated into French and German, which would help the understanding of students. Although the chapters are related, they can be read essentially independently.

All in all, this is a well-written text that I am happy to have on my bookshelf. This book should belong to any serious university physics library. It contains more than enough material for an advanced course on solid-state physics (from an experimental perspective) and will certainly be useful for private study as well. 